\title{
Introduction : les discours du tourisme, un objet privilégié pour l'analyse du discours
}

Introduction: Why Study the Discourses of Tourism?

\section{Galia Yanoshevsky}

\section{(2) OpenEdition}

\section{Journals}

\section{Electronic version}

URL: https://journals.openedition.org/aad/5425

DOI: 10.4000/aad.5425

ISSN: 1565-8961

\section{Publisher}

Université de Tel-Aviv

\section{Electronic reference}

Galia Yanoshevsky, "Introduction : les discours du tourisme, un objet privilégié pour l'analyse du discours", Argumentation et Analyse du Discours [Online], 27 | 2021, Online since 14 October 2021 connection on 16 October 2021. URL: http://journals.openedition.org/aad/5425; DOI: https://doi.org/ $10.4000 /$ aad. 5425

This text was automatically generated on 16 October 2021 .

\section{(c) (i) () $\Theta$}

Argumentation \& analyse du discours est mis à disposition selon les termes de la licence Creative Commons Attribution - Pas d'Utilisation Commerciale - Pas de Modification 4.0 International. 


\title{
Introduction : les discours du tourisme, un objet privilégié pour l'analyse du discours
}

Introduction: Why Study the Discourses of Tourism?

\author{
Galia Yanoshevsky
}

\section{Introduction ${ }^{1}$}

1 Les discours du tourisme apparaissent comme un objet privilégié pour les analystes du discours. Ils sont en effet traversés par des problématiques qui sont monnaie courante dans le domaine : subjectivation/objectivation, construction d'ethos, questions de genre ou étude des types de discours. On parlera des discours du tourisme, au pluriel, et non pas du discours du tourisme : il s'agit en effet d'un domaine à multiples acteurs, les touristes, les voyagistes, les ministères de tourisme et de patrimoine, les industries dérivées du tourisme - qui proviennent de différents horizons : l'économie, la culture, la valorisation du patrimoine, la publicité, la communication, la géographie, qui relèvent donc de régimes discursifs différents.

Étudier les discours du tourisme, c'est d'abord adhérer au paradigme foucauldien selon lequel comprendre un domaine c'est connaitre ses discours. Nous partons de l'idée que "le tourisme » est une «formation discursive " (Foucault 1969), donc un ensemble de textes aux horizons génériques différents, qui nous instruisent sur la situation, le contexte et les identités des acteurs de l'industrie du tourisme - producteurs, consommateurs, auteurs et promoteurs ${ }^{2}$. À partir du postulat que les discours sont révélateurs d'un domaine et de ses règles ou d'un pan de réalité (Amossy 2010a), nous étudions la chose touristique en fonction de ses manifestations discursives: ses textes, ses interactions. Mais les discours du tourisme sont aussi une construction du chercheur qui dans ce numéro explore les discours sur le tourisme, susceptibles de nous renseigner sur «la chose touristique» de façon directe - par le discours scientifique qui étudie les phénomènes sociolinguistiques du tourisme - ainsi que de 
manière indirecte - à travers le discours littéraire, qui mets en scène l'univers touristique, ses discours et leurs supports.

3 Les discours sont hybrides ; ils vont du discours de l'information et de la promotion aux discours critiques/axiologiques, qui fonctionnent dans la distance qui se creuse entre l'espace imaginé et l'espace visité. Le protagoniste principal des discours du tourisme, et sur le tourisme, est le voyageur-touriste, qui se voit à la fois loué et décrié. Souvent pensé comme un anti-héros du voyage (Urbain 1991), il est en même temps un produit et un producteur des discours, le consommateur et le consommé. Son corps participe pleinement à l'expérience ; mais l'expérience sensorielle et psychologique du touristevoyageur, bien que racontée dans les récits de voyages et notamment par des écrivains, n'est pourtant pas toujours exprimée dans les guides et il faut attendre les discours critiques, scientifiques ou littéraires pour qu'elle soit évoquée dans toute son ampleur. Les discours du tourisme jouent donc dans la zone du dit et du non-dit. C'est au touriste de faire le lien et d'activer l'information fragmentaire du guide - c'est ce que montrent les recherches qui se penchent sur les discours du guide et les usages de celui-ci. Qui plus est, le touriste est à la fois lecteur et auditeur - et son expérience ne se résume pas toujours au voyage lui-même et à l'orientation dans l'espace visité. Elle comprend aussi la lecture du guide, l'écoute de la radio et le voyage imaginaire - on peut voyager depuis son fauteuil. Étudier les discours du tourisme, c'est donc décortiquer ces situations d'énonciation et leurs scénographies.

4 Mais l'expérience touristique ne se limite pas à la quête individuelle ou singulière: derrière les quêtes personnelles se profile le collectif - on voyage, on fait le touriste, on rencontre l'autre, on découvre une autre culture, mais on rencontre aussi sa propre culture grâce au miroir tendu par la culture autre qu'on visite. Or, cette rencontre est conditionnée par les clichés, les stéréotypes et les idées reçues (Amossy 1982, 1991, 1997, Boyer 2007) réciproques de la culture visitante et de la culture visitée (Jaworski et Thurlow 2010). Ces stéréotypes jouent par ailleurs un rôle positif dans la possibilité de donner un sens au monde et à l'univers culturel visité. Aussi, dans le processus de la visite, on est soumis à ce qu'il faut faire et voir (Koshar 1998), aux injonctions de la publicité et de la promotion du patrimoine de l'autre. On est aussi confronté à sa propre culture et à des objectifs qui relèvent de ses propres besoins. Ainsi, par exemple, pour le pèlerin, le voyage en Terre Sainte favorise sa propre mobilité sociale : l'accomplissement du voyage est un signe de réussite sociale (comme le montre Sela, dans ce numéro). Mais visiter une autre culture peut aussi être perçu comme une intervention violente, la colonisation d'un espace autre, ce que montrent en effet les dernières tendances des études sur le tourisme (par ex. Rodolphe 2017). Étudier les discours du tourisme, c'est donc étudier le déploiement et le fonctionnement des idées reçues et des stéréotypes dans les propos des touristes et pour les touristes.

5 Si les acteurs du tourisme sont l'autre qu'on rencontre et avec qui on est censé interagir et converser (Jaworski et Thurlow 2010), ce sont aussi des espaces géographiques comme les villes et les pays. Ceux-ci font l'objet d'un marketing, leur patrimoine est marchandisé, leur image façonnée et refaçonnée par des imaginaires qui dépassent les imaginaires nationaux (Wilson dans ce numéro). Au vu de ces acteurs, étudier le tourisme c'est explorer les discours des protagonistes - les interactions verbales du touriste avec ses interlocuteurs dans le pays visité, mais aussi les discours de marketing des lieux visités. 
Ce numéro fournit d'abord un aperçu non exhaustif des études qui ont traité des discours du tourisme dans les deux dernières décennies, dans les domaines anglophone et francophone, puis se focalise sur la perspective prônée ici et ses postulats. Enfin, la présentation des articles du dossier met l'accent sur la variété des corpus et des approches analytiques fondées sur un examen du discours en en dégageant quelques axes communs.

\section{Un état du champ (non exhaustif) des approches discursives du tourisme dans les deux dernières décennies}

7 Un aperçu des recherches combinant l'analyse du discours et la linguistique de corpus avec les études sémiotiques des deux dernières décennies en montre l'intérêt pour l'étude du tourisme. Dans le domaine anglophone, plusieurs recherches mettent l'accent sur ces approches. Comme son nom l'indique, l'ouvrage collectif édité par Jaworski et Pritchard (2005), Discourse, Communication and Tourism, se concentre sur le discours du tourisme en arguant que le tourisme est un champ d'enquête fécond pour les sciences humaines et sociales. À partir de corpus divers comme des programmes télévisés, des messages en ligne, des cartes postales, des brochures et des publicités, ce collectif décrit et analyse les pratiques touristiques et les interactions entre hôtes et touristes dans diverses communautés de voyages : organisés, routards, indépendants, ainsi que divers types de tourisme (écotourisme, agrotourisme, de patrimoine ou encore ethnotourisme). Les contributeurs du volume, spécialistes de différentes disciplines (dont la sociologie, la linguistique, les médias et les arts du spectacle, les études de communication), prennent le discours dans trois sens. D'abord, comme communication verbale, ensuite comme usage de la langue dans une situation de communication et dans un contexte culturel et social donné, enfin, comme activité idéologique qui façonne la réalité sociale, les identités et les relations avec les autres, voire génère des modèles de pouvoir, de domination et de contrôle ${ }^{3}$. Ce sont les deux derniers aspects du discours qui sont pris en considération par Thurlow et Jaworski, qui, dans Tourism Discourse (2010), offrent de nouvelles perspectives sur le rôle des discours oraux, écrits et visuels dans la représentation et la production du tourisme en tant qu'industrie culturelle mondiale. Afin d'examiner l'interaction entre les ordres symboliques et économiques de la mobilité mondiale, leur analyse est fondée sur des études empiriques de genres touristiques clés, comme les émissions télévisées de vacances, les journaux de voyage, les glossaires de guides, les cartes postales de vacances et les magazines de bord. Combinant la linguistique de corpus et l'analyse du genre discursif, ils étudient les modalités selon lesquelles les discours du tourisme reflètent de manière dynamique de nouvelles tendances sociétales comme la mobilité globale et sa contribution au développement de l'industrie du tourisme. Dans Tourism discourse: Languages and banal globalization (2011), ils cherchent à apporter une perspective de sociolinguistique ou d'analyse du discours au domaine des études touristiques : leur travail se concentre sur le rôle de la langue et de la communication dans le domaine. De même que le tourisme s'est avéré être un thème de recherche important pour les anthropologues et les sociologues, il est un objet-clé pour l'étude des processus humains de communication - notamment en ce qui concerne les contacts 
et les échanges interculturels, mais aussi en termes de circulation de "matériel» linguistique (par exemple les genres, les discours et les styles).

Hannam et Knox (2015) quant à eux, distinguent entre différentes méthodes d'analyse pour les études de tourisme (tourism studies) - l'analyse de contenu, l'analyse textuelle simple, l'analyse sémiotique et les approches poststructuralistes d'analyse du discours ; elles portent sur des corpus variés comme les documents écrits et les matériaux visuels (photographies et brochures) afin de fournir un cadre critique à d'autres chercheurs. Visant un public intéressé par le discours sur le tourisme d'un point de vue sociosémiotique et discursif, Maci (2020) argue que l'industrie du tourisme développe différents genres afin de vendre le même produit à des cibles différentes et sur des marchés différents. En combinant la linguistique de corpus et l'analyse de genre, elle suggère que le discours touristique semble avoir développé de nouvelles stratégies linguistiques à la fois spécialisées et promotionnelles, caractérisées par l'émergence d'un nouveau mode de communication hypertextuel décrivant la destination sur le mode euphorique et véhiculant l'idée de responsabilité du touriste vis-à-vis de ses choix de destination.

9 Fortes de l'approche critique de l'analyse du discours (la CDA) et d'un logiciel d'analyse de corpus, de Carvalho Figueiredo et Alvares Pasquetti (2016) explorent les mécanismes du guide en ligne Best in Travel 2015 : Top 10 cities et sa traduction en brésilien, tous deux publiés en ligne en 2014 par Lonely Planet. L'étude vise à explorer certaines des caractéristiques du discours touristique actuel en analysant le réseau de personnes et de pratiques impliquées dans ces publications, leurs caractéristiques textuelles et leurs images. Les chercheuses définissent à partir de là le type de public auquel s'adressent ces guides et notamment un public familier de la culture numérique, doté d'un pouvoir d'achat et manifestant une prédilection pour les produits fétiches ou " gourmets".

Enfin, le collectif dirigé par Bielenia-Grajewska et Cortes de los Rios (2017) - Innovative Perspectives on Tourism Discourse - présente les avancées de la recherche sur le rôle du langage dans l'industrie du tourisme et du voyage, et couvre des domaines relatifs au tourisme comme la communication interculturelle, les voyages d'aventure et le marketing touristique. Consacrée aux Discourse Studies, la première partie du volume met l'accent sur le numérique - à savoir les sites web multilingues consacrés aux différents secteurs du tourisme comme les loisirs, les mobilités sportives, et les services pour visiteurs handicapés, ainsi que sur différents aspects comme la durabilité, l'hypermobilité, la localisation et les techniques d'extraction des données des sites web consacrés au tourisme. Les méthodes employées proviennent de l'analyse multimodale, de l'analyse des interactions verbales et de l'analyse numérique des documents textuels (Rhetorical Structure Theory [RST] et Universal Networking Language [UNL]).

11 Bien que moins abondantes dans le domaine francophone ${ }^{4}$, il existe quelques études du tourisme qui partent de postulats discursifs. Le collectif de Baider, Burger et Gutsos (2004) rassemble des chercheurs francophones et anglophones de différents horizons théoriques dans une perspective pluridisciplinaire en combinant à la fois l'analyse du discours et la sémiotique, les théories post-coloniales et les théories féministes. La communication touristique y est considérée comme comportant une triple dimension discursive, sociale et culturelle. Le volume aborde l'identité discursive des acteurs du tourisme et examine les genres du discours touristique comme le guide et la brochure en étudiant comment ces phénomènes sont exploités de manière distincte par 
différentes communautés linguistiques, sociales et culturelles « qui 'vendent' l'altérité touristique » (ibid.).

12 Ainsi, la deuxième partie propose des analyses de guides de voyage dans la perspective de l'analyse du discours en s'attardant sur l'usage des stéréotypes dans un contexte de représentations identitaires dans les genres phares de l'industrie touristiques: les guides de voyage et les publicités des voyagistes ${ }^{5}$. La troisième partie du volume aborde la dimension sémiotique et communicationnelle du discours touristique. Cette perspective se focalise sur la fonction des textes et images touristiques considérés comme des représentations culturelles et des " agents " sémiotiques engagés dans un processus complexe au gré duquel des identités sont construites et maintenues par et dans la communication.

13 Support privilégié de l'industrie du tourisme, le guide touristique a fait l'objet de nombreuses études. Dans une perspective historique et de patrimoine, le numéro spécial de la revue Situ dirigé par Cohen, Toulier et Vajda (2011) ainsi que la bibliographie indicative des guides de voyages et de tourisme sur deux décennies (1990-2010) par Évelyne Cohen (2011) restent des références majeures. Il a provoqué aussi l'intérêt de chercheurs en analyse du discours comme Moirand (2004, dans le volume de Baider et al.) et Seoane (2013), qui a consacré un volume, issu d'une thèse de doctorat en sciences du langage, aux guides touristiques français (le Guide du Routard et le Guide Gallimard). Seoane y étudie les mécanismes énonciatifs dans les guides touristiques: les genres, les positionnements énonciatifs et notamment l'ethos de l'instance énonciatrice (Amossy 2010b).

14 Dans la tradition de la sémiotique communicationnelle, (suivant Barthes 1957 et Gritti 1967), Yves Jeanneret (2014) étudie le guide touristique ou " objet écrit pragmatique ", en prenant en considération ses dimensions matérielles, visuelles et éditoriales (131-132) : «l'écrit se regarde et se manipule, il est affaire de supports, de rythmes et de formes. » (132). C'est pourquoi l'approche discursive doit être secondée d'une approche sémiotique. "D'autre part, cet écrit n'est pas seulement destiné à être lu mais aussi pratiqué »(ibid). Dans cette tradition, Vergopoulos (2010) soutient une thèse de doctorat où elle se propose d'étudier les légendes comme mode opératoire des guides touristiques ${ }^{6}$. Par la façon dont elles sont commentées et mises en œuvre dans et par les guides, les légendes sont un objet de discours capable d'instituer un rapport à la culture qui serait de l'ordre de la curiosité ou de l'insolite en renversant ou détournant l'ordre culturel.

Les études sur les imaginaires touristiques qui combinent des perspectives anthropologiques et culturelles (Salazar et Graburn 2014) et qui ont pour souci le patrimonial (entre autres Gravari-Barbas et Graburn 2012) sont complétées par le volume de Raus, Cappelli et Flinz (2017) consacré au guide touristique comme lieu de rencontre entre un dispositif pluri-sémique (liant texte et image) et le patrimoine culturel. Yanoshevsky (2017) y étudie l'hybridation générique d'un album de photos qui se veut guide touristique, tout en montrant, chemin faisant, le lien du guide avec la construction d'un ethos national.

16 Enfin, les aspects de globalisation, traités amplement dans les études anglophones, sont explorés dans des études en sciences du langage en France. Ainsi, par exemple, Wilson (2016) étudie les dynamiques sociales de Marseille à travers les usages langagiers de l'office de Tourisme et des Congrès de la ville, et conclut que des «traces laissées sur le 
tissu sociolinguistique de la ville " se dégagent des inégalités liées aux processus de globalisation de la ville.

\section{Notre perspective : Étudier les discours du tourisme c'est...}

Compris au sens large du terme, le texte (qu'il soit verbal, visuel ou interactionnel) ne serait pas une simple représentation du monde : il participe pleinement à sa création. Le langage serait ainsi la matière même qui construit l'univers du tourisme et ses pratiques (Thurlow et Jaworski 2010 et 2011, Baider, Berger et Goutsos 2004). Les éléments langagiers et leur mise en discours façonnent notre manière de voir, de visiter, d'occuper et de comprendre l'espace touristique.

Étudier les discours du tourisme, c'est alors explorer la mise en mots (Koren 1996), la mise en tourisme de la destination (Seoane 2013) ${ }^{7}$ ou encore la mise en littérature de l'expérience touristique et du patrimoine (Delaitre et Farina et Sini dans ce numéro). Par «discours du tourisme" nous entendons aussi bien les discours au profit des touristes (discours promotionnels, des voyagistes, des services pour touristes, discours sur le patrimoine, sur les espaces géographiques visités) que les discours produits par les sujets de l'expérience touristique (les consommateurs de l'expérience touristique à savoir les touristes). Nous entendons aussi par-là l'univers des signes - les représentations graphiques et visuelles de l'espace parcouru par le touriste - que ce soient les indications dans les guides (les cartes, les indications de routes, de parcours...) ou les panneaux dans les espaces consacrés au passage des touristes comme les aéroports (Thurlow et Jaworski 2010). Ces discours à la fois descriptifs et prescriptifs (What ought to be seen, Koshar 1998) sont donc la matière première de l'analyste du discours. Celle-ci s'intéressera aussi à l'univers des objets, compris comme texte ${ }^{8}$, qui expriment de façon symbolique l'expérience du vécu touristique (comme les pins, les souvenirs), et qu'il étudiera par des méthodes empruntées à la sémiotique (Culler 1981, Jeanneret 2015, Souchier et Wrona 2015) 9 .

19 Étudier les discours du tourisme, c'est aussi explorer les métadiscours, à savoir les discours critiques ou érudits sur l'expérience touristique et son industrie : c'est d'abord ce qu'observent les chercheurs prônant l'approche de la CDA (Critical Discourse Analysis) sur les liens qui existent entre tourisme et colonialisme, globalisation de l'industrie du tourisme et exploitation du monde en voie de développement (Thurlow et Jaworski 2010 et 2011) ${ }^{10}$. C'est aussi ce qu'explore le discours littéraire qui met en scène des voyageurs-touristes dans des lieux touristiques comme les plages populaires, les villages de vacances, les îles magiques et ainsi de suite, et qui adopte une distance critique vis-à-vis des pratiques sociales et des rites associés au tourisme. Ainsi, plusieurs écrivains comme Michel Houellebecq, Marie Ndiaye, Lydie Salvayre (comme le mentionne Delaitre dans ce numéro) se sont penchés sur l'industrie du tourisme, les touristes et leurs comportements. Étudier les mécanismes de ces discours critiques qui font apparaître une polyphonie de voix exposent l'industrie et les acteurs touristiques est l'une des tâches de l'analyste du discours désireux de sonder l'objet d'étude «tourisme $»^{11}$.

Étudier les discours du tourisme, c'est aussi explorer ses « canaux de transmission »ses genres, aussi bien que ses supports matériels : les guides touristiques, les catalogues 
de voyages, les brochures de publicité et de commercialisation des lieux, les sites internet des voyagistes, mais aussi les cartes postales, les cartes géographiques et les panneaux des sites, la télévision et la radio. Dès lors, on prête attention non seulement au dit, mais aussi au "comment-dire ", au contexte de l'énonciation - symbolique ou matériel. Ce n'est pas uniquement le contenu du panneau indiquant le chemin ou le site qui importe, mais aussi sa disposition dans l'espace et sa composition matérielle qui conditionne le regard du touriste et du visiteur (Thurlow et Jaworski 2010, Noy 2016). Il en va de même pour le passage entre les indications dans les manuels du tourisme et ce qu'on aperçoit lorsqu'on se rend sur un site (Cohen-Hattab 2004, Jeanneret 2015, Vergopoulos dans ce numéro). Enfin, c'est la manière dont l'expérience et les imaginaires touristiques sont transmis par le travail imagé ou sonore des supports matériels, et notamment par l'espace sonore de la radio (Equoy-Hutin dans ce numéro). Le guide touristique figure parmi les supports matériels qui ont le plus retenu l'attention des chercheurs du tourisme qui s'intéressent aux problématiques du patrimoine (Cohen, Toulier et Vajda 2011) ou à l'imaginaire national (Yanoshevsky 2017). Outre sa fonction d'orientation du touriste, le guide touristique participe à la construction de l'identité de la culture visitée et de la culture visitante (Buzard 1993, Koshar 1998, Furlough 2002, Baider, Burger et Goutsos 2004, Hallet et Kaplan-Weinger 2010). Il est également le support matériel des imaginaires touristiques (Salazar er Graburn 2014 : 12) participant à la création des mythes touristiques (Selwyn 1996, Leite dans Salazar et Graburn $2014: 265$ ). Mais du fait de son hybridité générique, le guide a attiré aussi les analystes du discours: entre le promotionnel et l'informationnel, le guide a des visées descriptives, prescriptives et critiques (Kerbrat-Orecchioni 2004) ou encore didactiques (Moirand 2004). Seoane (2013) nous invite à étudier différents guides par rapport à leurs horizons d'attente (le Guide du Routard, le Guide Bleu...). Chaque guide est ainsi contextualisé, en fonction de sa périodicité, ses producteurs et ses consommateurs. Ainsi, les divers guides français à destination d'Israël montrent la coexistence et la confrontation des dimensions singulières et collectives comme un trait constitutif du genre : l'auteur du guide (locuteur tantôt individuel, tantôt collectif) peut jouer plusieurs rôles et entrecroiser des points de vue divers : le sien, celui des visiteurs et celui des visités (Yanoshevsky 2020). Ailleurs Seoane et d'autres chercheurs montrent la contamination réciproque entre différents dispositifs communicationnels du guide: les supports papier et les sites en ligne (Seoane 2013, Stoian 2017).

22 Étudier les discours $\mathrm{du}$ tourisme, c'est explorer les identités propres à la communication touristique : notamment celle du "voyageur » ou du « touriste »; celle du « voyagiste » ou du " publicitaire »; celle de l'« hôte » ou de l'« autochtone ». Toutes ces identités ont ceci en commun d'appartenir au dispositif discursif de la communication touristique (Baider et al. 2004). Elles ne sont pas fixes, mais évoluent dans l'interaction avec l'autre, et dans l'expérience touristique subie par le voyageur en vue du contact avec le lieu visité (Sela dans ce numéro). On devient colonisateur à partir du moment où s'instaurent des rapports d'inégalité avec les locaux, mais ces rapports de forces peuvent aussi être inversés lorsque les locaux se sentent supérieurs aux visiteurs (Thurlow et Jaworski 2011). La nature dynamique des identités se reflète également dans la transformation que subissent les pèlerins en contact avec leur lieu de visite et qui exerce même une influence sur leur statut social lorsqu'ils rentrent chez eux, et contribuent à la création d'une identité collective (Sela dans ce numéro). Ces identités se manifestent aussi dans la production d'une impression de famille dans le 
tourisme diasporique, lorsque par exemple les Louisianais se rendent en Acadie (Bruce et Urbain dans ce numéro). Enfin, la notion d'identité n'est pas réservée aux seuls acteurs, mais se réfère aussi aux espaces géographiques et touristiques et à la manière dont leur image de marque est formée et transformée au gré des imaginaires qui leur sont attachés (Debarbieux 2003, Davoudi 2018, Wilson dans ce numéro).

\section{Les articles de ce numéro - variété des corpus et d'approches d'analyse discursive, et axes communs}

Issues d'horizons théoriques différents (les sciences du langage, l'anthropologie, la sociologie, les études de communication/des média, la littérature, la linguistique, la géographie du tourisme), et des thématiques/corpus les plus divers - le pèlerinage (Tal Sela), le tourisme diasporique (Clint Bruce et Emilie Urbain), les émissions de tourisme à la radio (Séverine Equoy-Hutin), les romans du tourisme de Houellebecq (Carole Delaitre), une base de données comportant le lexique des biens culturels des voyageursécrivains (Annick Farina et Lorella Sini), l'image de marque des villes touristiques (Adam Wilson) et le corps dans le guide touristique (Hécate Vergopoulos) - ces recherches ont toutes ceci en commun, qu'elles se penchent sur les discours $d u$ tourisme. Elles analysent les genres, les modes d'énonciation et les énonciateurs, les supports et les contextes d'énonciation. Et ce, par des méthodes variables, allant de l'analyse du lexique (Farina et Sini), de l'argumentation (Bruce et Urbain, Equoy-Hutin) et de la rhétorique verbale, visuelle (Sela, Vergopoulos) et sonore (Equoy-Hutin), de la construction d'image (Equoy-Hutin, Wilson), des stéréotypes et des lieux communs (Delaitre, Equoy-Hutin, Farina et Sini, Bruce et Urbain), des interactions verbales (Bruce et Urbain, Equoy-Hutin), des analyses sémio-discursives prenant en compte les supports du discours touristiques (guides, brochures, pins, émissions radiophoniques), leur matérialité et leurs fonctions (Sela, Vergopoulos)

Malgré cette diversité d'approches théoriques, des thématiques et des corpus, trois axes majeurs se dégagent des sept articles qui composent le dossier.

\subsection{L'imaginaire touristique}

Qu'il soit lié au patrimoine, à la marchandisation ou au type de déplacement, qu'il soit exprimé par des supports et des contextes énonciatifs et génériques aussi différents que la littérature (romans, récits de voyage), les guides et brochures touristiques, les interactions sur site, ou encore la radio, qu'il soit articulé avant, pendant ou après le voyage, l'imaginaire touristique est omniprésent. Il est bien le thème principal de l'expérience touristique. Il transparaît d'abord dans l'activité économique de marketing, qui consiste à construire, comme nous le montre Adam Wilson, des images de ville liée à des identités supranationales (européenne pour Metz, méditerranéenne pour Marseille). Cet imaginaire se manifeste aussi dans la rencontre singulière du touriste voyageur avec le lieu qu'il visite et les liens qui se tissent entre son identité (ethnique, religieuse) et les images qui lui sont offertespar les organismes organisateurs du voyage : c'est le cas, décrit par Tal Sela, du pèlerinage en Terre Sainte de l'Afrique subsaharienne et la possibilité de mobilité sociale qu'elle représente pour ses participants. C'est aussi, comme le montrent Clint Bruce et Emilie Urbain, l'expérience vécue par les Louisianais qui se rendent dans leur terre ancestrale d'Acadie 
lors du Congrès Mondial Acadien, et qui visitent des sites patrimoniaux où se produit un effacement de la distance mémorielle et géographique grâce aux éléments doxiques d'une identité partagée qui leur permet de se sentir chez eux, alors qu'ils sont ailleurs. C'est enfin ce que dégage la recherche lexicologique menée par Annick Farina et Lorella Sini, sur un corpus de récits de voyage d'écrivains francophones, principalement du $19^{\mathrm{e}}$ siècle, à Florence, berceau du Rinascimento : si l'écrivain réalise un voyage singulier, en visitant les « haut-lieux » du parcours typique de son époque, il n'empêche qu'il est à la fois un "testeur» des lieux communs (dans les deux sens du terme: des lieux physiques patrimoniaux et l'idée du voyage typique) ainsi que leur promoteur: il participe ainsi à la production et au maintien de l'imaginaire touristique dans la littérature.

\subsection{Le singulier et le banal de l'expérience touristique}

Ce que révèlent aussi les études du dossier, c'est la tension inhérente à l'expérience touristique entre la singularité (ou la subjectivité) du voyage et l'objectivisation des parcours proposés selon des sentiers battus, balisés de clichés. Cette rencontre a lieu souvent dans le contact que maintient le touriste-voyageur avec le guide, ce support par excellence de l'activité touristique, dans une situation d'énonciation où il est à la fois lecteur et acteur, performant les parcours proposés. Ce que nous montre Hécate Vergopoulos, à partir d'un corpus de guides culturels et pratiques de New York, c'est le travail accompli par ce corps voyageur, un travailleur qui a volontairement déblayé un espace-temps continu de son quotidien pour faire usage de son pouvoir d'achat ailleurs et massivement. La singularité du parcours individuel se voit alors annexée au tourisme de masse et aux règles du marché de l'industrie touristique. Mais l'énonciation touristique n'est pas réservée aux seuls genres routiniers du tourisme (Maingueneau 2004): elle se manifeste aussi dans les genres auctoriaux. Si les écrivains y interviennent, c'est d'abord parce qu'eux aussi sont des touristes. Ainsi, les écrivainsvoyageurs décrivent les « hauts-lieux » de Florence en définissant le "beau », mais en même temps expriment, comme le montrent Farina et Sini, un désenchantement face à la banalité de leur rencontre avec les œuvres-clé du patrimoine de la Renaissance. La collision entre la singularité et le caractère banal de l'expérience touristique se manifeste aussi dans la critique développée dans les romans touristiques de Houellebecq. À travers une analyse de leur polyphonie énonciative (comportant les voix d'un énonciateur narrateur, des personnages et occasionnellement de la voix auctoriale), Carole Delaitre montre la façon dont Houellebecq critique le tourisme par un discours qui pastiche et parodie les discours des guides semés de stéréotypes et de clichés, mais qui conseille d'éviter les lieux banals que croisent les touristes sur leur chemin, ces lieux de l'industrie métallurgique, productrice pourtant des véhicules (chemins de fer, automobiles) qui permettent le déplacement du touriste vers sa destination...

\subsection{Expérience sensorielle et expérience collective}

Ce que montrent enfin les analyses des discours de ce dossier, c'est à quel point l'expérience sensorielle - les images, les sens, la présence corporelle (Bernard 1995, Le Breton 2008) - est décisive dans la pratique touristique qui se déroule dans la zone entre le singulier et le collectif (Paissa et Koren 2020, Amossy et Orkibi 2021). Comment 
rendre sensibles les auditeurs de la radio aux lieux et aux images par la sonorité et par le langage radiophonique? C'est la question que se pose Séverine Equoy-Hutin qui travaille sur des extraits du magazine de tourisme radio Et si on partait? traitant deux destinations (L'Aveyron et la Mongolie). Il s'avère que le touriste est d'abord un auditeur, sensibilisé à l'expérience touristique grâce à l'expertise des animateurs et à l'hyper-structure sonore de l'émission (musique et bruits). Ce corps sensibilisé, nous montre Hécate Vergopoulos, est aussi un corps en mouvement, fatigué par la réalisation du parcours intense proposé par le guide touristique de court séjour. Mais la lassitude ne se restreint pas au seul domaine physique car elle a son équivalent mental. Provoquée chez l'écrivain-voyageur par une rencontre impressionnante ou bien décevante avec un objet de patrimoine mille fois décrit, elle peut se transformer en mal mental tel le "syndrôme de Stendhal ", trouble émotionnel qui mobilise une émotion profonde mais qui est probablement aussi à la base de la construction d'un ethos d'écrivain-voyageur dans les villes d'art imprégnées d'icônes culturelles, voire de symboles mystiques, et où l'écrivain «n'hésite pas à invoquer ce topos, à témoigner de l'extraordinaire qui enivre et qui transporte » (Farina et Sini).

L'émotion profonde participe aussi à la formation d'un ethos collectif entendu dans le sens de fonction identitaire (Amossy et Orkibi 2021: 11). Il s'agit de procurer un sentiment d'appartenance en permettant l'identification à une image porteuse. Ainsi, un ethos de groupe est instigué par une émotion profonde provoquée chez les touristes louisianais en visite dans les lieux ancestraux. Soumis à l'immersion sensorielle et mémorielle lors de la visite, ils expriment un sentiment de "retour à la famille ", comme le montrent Bruce et Urbain. Enfin, l'émotion ressentie par le pèlerin individuel en contact avec la Terre Sainte est exploitée dans les photos de groupe. Ces gestesclichés de la visite en plus de ses objets dérivés comme les pins et les diplômes distribués en fin de parcours sont comme l'incarnation de l'expérience personnelle et contribuent à l'ethos collectif du pèlerin, dans le sens constitutif du terme : il s'agit de "faire exister un groupe en le manifestant aux yeux des autres" (Amossy et Orkibi 2021 : 21). Le pèlerin peut s'élever dans l'échelle sociale grâce au prestige octroyé par les manifestations matérielles du pèlerinage (Sela).

\section{Conclusion}

Dans le prolongement des travaux en analyse des discours du tourisme, ce numéro montre l'intérêt réciproque que présente le tourisme pour les analystes du discours, et les travaux qui explorent les mécanismes discursifs pour les chercheurs dans tous les domaines du tourisme - géographie du tourisme, études du patrimoine, économie du tourisme, ethnographie, sociologie, histoire.... Pour les analystes du discours, c'est un objet de rêve car s'y télescopent des phénomènes discursifs qui lient le discours au social: l'image du touriste, des voyagistes, les genres discursifs routiniers et auctoriaux, l'hybridité générique, la scène d'énonciation, l'ethos singulier et collectif... Pour les chercheurs qui se penchent sur le tourisme, les analyses discursives exposent et répertorient des phénomènes qui restent inaperçus dans les études qui négligent la micro-analyse et ne se nourrissent pas de ses apports.

Le présent numéro a été conçu lors d'une période de transformation massive dans l'industrie du tourisme causée par le COVID-19. Trop proche de l'événement, il n'explore pas les tendances actuelles à la lumière des contraintes de mobilité imposées 
par la pandémie, comme le «stay-cation » (littéralement les vacances chez soi, ou les vacances sans déplacement). Il ne parcourt pas non plus des thèmes qui ont pris de l'ampleur en raison de l'arrêt quasi-total à l'échelle mondiale de l'industrie touristique entre 2019-2020. Ainsi, aux vocables connus déjà avant le COVID, comme «tourisme écologique ", «tourisme durable" ou encore «tourisme comme colonialisme ${ }^{12}$ " s'ajoutent d'autres, comme le "surtourisme", ou les effets du tourisme de masse sur des lieux surexploités avant la pandémie (Gravari-Barbas 2021). Repérer ces nouveaux termes et étudier les discours où ils figurent pourra nous éclairer sur les tendances actuelles du tourisme et de ses pratiques.

\section{BIBLIOGRAPHY}

Amossy, Ruth \& Elisheva Rosen. 1982. Les Discours du cliché (Paris : CDU et SEDES)

Amossy, Ruth \& Anne Herschberg Pierrot. 1997. Stéréotypes et clichés - Langue, discours, société (Paris : Nathan)

Amossy, Ruth. 1991. Les idées reçues. Sémiologie du stéréotype (Paris : Nathan)

Amossy, Ruth, 2010a. L'argumentation dans le discours (Paris : A. Colin)

Amossy, Ruth. 2010b. La Présentation de soi. Ethos et identité verbale (Paris : PUF)

Baider, Fabienne, Marcel Burger \& Dionysis Goutsos (éds). 2005. La communication touristique.

Approches discursives de l'identité et de l'altérité (Paris : L'Harmattan)

Barthes, Roland. 1957. Mythologies (Paris : Seuil)

Bernard, Michel. 1995. Le Corps (Pairs : Seuil)

Bielenia-Grajewska, Magdalena \& Enriqueta Cortes de los Rios (éds). 2017. Innovative Perspectives on Tourism Discourse, IGI Global

Boyer, Henri (éd.). 2007.Stéréotypage, stéréotypes : fonctionnements ordinaires et mises en scène, vol. 1 Média(tisation)s(Paris : L'Harmattan)

Buzard, James. 1993. The Beaten Track. European Tourism, Literature, and the Ways to 'Culture', 1800-1918 (Oxford : Oxford U. P.)

Cohen, Évelyne \& Bernard Toulier. 2011. «Les guides de tourisme, un patrimoine et un objet d'étude », Situ 15 [En ligne] http://journals.openedition.org/insitu/723

Cohen, Évelyne, Bernard Toulier \& Joanne Vajda (éds). 2011. « Le patrimoine des guides : lectures de l'espace urbain européen », Situ 15, 292-355

Cohen-Hattab, Kobi. 2004. «Zionism, Tourism, and the Battle for Palestine: Tourism as a PoliticalPropaganda Tool », Israel Studies $9: 1,61-85$

Culler, Jonathan. 1981. « The Semiotics of Tourism », American Journal of Semiotics $1: 1,127-140$

Davoudi, Simin. 2018. « Imagination and spatial imaginaries: a conceptual framework », Town

Planning Review 89, 97-107

Argumentation et Analyse du Discours, 27 | 2021 
Debarbieux, Bernard. 2003. «Imaginaire géographique », Lévy, Jacques \& Michel Lussault (éds). Dictionnaire de la géographie et de l'espace des sociétés (Paris : Belin), 489-491

De Carvalho Figueiredo, Débora \& Camila Alvares Pasquetti. 2016. « The discourse of tourism: an analysis of the online article "Best in Travel 2015: Top 10 cities" in its translation to Brazilian Portuguese » Ilha do Desterro 69 : 1, 201-212 [En ligne] https://doi.org/ $10.5007 / 2175-8026.2016 v 69 n 1 p 201$

Équipe MIT (Équipe Mobilité, Itinéraire et Territoires). 2002. Tourismes 1, Lieux communs (Paris : Belin)

Foucault, Michel, 1969 L'archéologie du savoir (Paris : Gallimard)

Furlough, Ellen. 2002 « Une leçon des choses : Tourism, Empire, and the Nation in Interwar France », French Historical Studies $25: 3,441-473$

Gravari-Barbas, Maria \& Nelson Graburn. 2012. « Imaginaires touristiques », Via. Tourism Review 1 [En ligne] https://doi.org/10.4000/viatourism.1180

Gravari-Barbas, Maria. 2021. « Du surtourisme aux nouvelles formes de tourisme?», Cahiers français, Sept.-Oct., 38-47

Gritti, Jules. 1967. « Les contenus culturels du Guide Bleu : monuments et sites "à voir" », Communications 10, 51-64

Hallett, Richard W. \& Judith Kaplan-Weinger. 2010. Official Tourism Websites (Tourism and Cultural Change), (Bristol, Buffalo \& Toronto : Channel View Publications)

Hannam, Kevin \& Dan Knox. 2015. “Discourse Analysis in Tourism Research A Critical Perspective” Tourism Recreation Research $30: 2,23-30$

Jaworski, Adam, et Annette Pritchard (éds). 2005. Discourse, Communication, And Tourism (Clevedon : Channel View Publications)

Jaworski, Adam \& Crispin Thurlow. 2010. Semiotic Landscapes. Langage, Image, Space (New York: Continuum)

Jeanneret, Yves. 2015. « Le guide touristique, un objet écrit pragmatique », dans Jean-Jacques Boutaud \& Karine Berthelot-Guiet, Sémiotique Mode d'emploi, (Lormont : Le bord de l'eau), 131-172

Kerbrat-Orecchioni, Catherine. 2004. «Suivez le guide ! Les modalités de l'invitation au voyage dans les guides touristiques : l'exemple de l'île d'Aphrodite ", Baider, Fabienne, Marcel Burger \& Dionysis Goutsos (éds). Identité et altérité : analyses discursives de la communication touristique (Paris : L'Harmattan), 133-150

Koren, Roselyne. 1996. Les enjeux éthiques de l'écriture de presse et la mise en mots du terrorisme (Paris : L'Harmattan)

Koshar, Rudy. 2008. « What Ought to Be Seen': Tourists' Guidebooks and National Identities, Le Breton, David. La Sociologie du corps (Paris : PUF)

Leite, Naomi. 2014. «Afterward. Locating Imaginaries in the Anthropologies of Tourism », Salazar, Noël B. \& Nelson H.H. Graburn (éds.). Tourism Imaginaries. Anthropological Approaches (New York, Oxford : Berghahn)

Maci, Stefania M. 2020. English Tourism Discourse. Insights into the professional, promotional and digital language of tourism (Italy: Hoepli) 
Maingueneau, Dominique. 2002. «L'ethos, de la rhétorique à l'analyse du discours » [En ligne] http://dominique.maingueneau.pagesperso-orange.fr/pdf/Ethos.pdf.

Maingueneau, Dominique. 2004. «Typologie des genres de discours institués » [Version remaniée des pages 180-187 du Discours littéraire (Paris : A. Colin)]

Maingueneau, Dominique. 2011. « Pertinence de la notion de formation discursive en analyse de discours ", Langage et société, 1 : 135, 87-99 [En ligne] https://www.cairn.info/revue-langage-etsociete-2011-1-page-87.htm

MIT (Équipe Mobilité, Itinéraire et Territoires). 2002. Tourismes 1, Lieux communs (Paris : Belin)

Moirand, Sophie. 2004. « Le même et l'autre dans les guides de voyage au XXI ${ }^{\mathrm{e}}$ siècle », Baider, Fabienne, Marcel Burger \& Dionysis Goutsos (éds). Identité et altérité : analyses discursives de la communication touristique (Paris : L'Harmattan), 151-172

Moïse, Claudine. 2011. «L'économie mondialisée et le tourisme : un domaine à explorer pour la sociolinguistique francophone? » Mondes du Tourisme 4, 4-17

Noy, Chaim. 2017. «Participatory Media and Discourse in Heritage Museums : Co-constructing the Public Sphere? », Communication, Culture \& Critique $10: 2,280-301$

Paissa, Paola \& Roselyne Koren (éds). 2020. Du singulier au collectif: construction(s) discursive(s) de l'identité collective dans le débat public (Limoges : Lambert-Lucas).

Raus, Rachele Gloria Cappelli \& Carolina Flinz (éds.). 2017. Le guide touristique : lieu de rencontre entre lexique et images du patrimoine culturel, Vol. 2, (Florence : Firenze U. P.)

Rodolphe, Christin. 2017, Manuel de l'anti-tourisme (Paris : Écosociété)

Salazar, Noel \& Nelson H. H. Graburn, (éds). 2014. Tourism Imaginaries, Anthropological approaches (New York \& Oxford: Berghahn)

Selwyn, Tom (éd.). 1996. The Tourist Image: Myths and Myth-Making in Tourism (Chichester : Wiley)

Seoane, Annabelle. 2012. Genre de discours et positionnements énonciatifs dans les guides touristiques : le Guide du Routard et le Guide Gallimard, Thèse de doctorat sous la direction de Dominique Maingueneau, Paris Est et Centre d'étude des discours, images, textes, écrits et communications (Créteil)

Seoane, Annabelle. 2013a. Les mécanismes énonciatifs dans les guides touristiques : Entre genre et positionnements discursifs (Paris : L'Harmattan)

Seoane, Annabelle. 2013b. « Les guides touristiques : vers de nouvelles pratiques discursives de contamination », Mondes du Tourisme 8, 33-43 [En ligne] http://journals.openedition.org/ tourisme $/ 81$

Souchier Emmanuel \& Adeline Wrona. 2015. «L'impensé du texte. Pour une approche sémiotique du texte entre "image du texte", rhétorique et médiation, Boutaud, Jean-Jacques \& Karine Berthelot-Guiet (éds). Sémiotique, mode d'emploi (Lormont : Le Bord de l'eau), 173-189

Stoian, Claudia Elena. 2017. « The Discourse of Tourism from a Systemic Functional Perspective: The Case of Tourism Websites ", Bielenia-Grajewska, Magdalena \& Enriqueta Cortes de los Rios (éds). Innovative Perspectives on Tourism Discourse, 181-200

Thurlow, Crispin \& Adam Jaworski. 2010. Tourism Discourse. Language and Global Mobility (Basingstoke/New York : Palgrave Macmillan)

Thurlow, Crispin \& Adam Jaworski. 2011. « Tourism discourse: Languages and banal globalization ", Applied Linguistics Review 2, 285-312 
Urbain, Jean-Didier. 1991. L'Idiot du voyage (Paris : Plon)

Vergopoulos, Hécate. 2010. Tourisme et curiosités : approche communicationnelle du légendaire dans les guides de voyage imprimés, thèse de doctorat, sous la direction de Yves Jeanneret, Université d'Avignon et Université du Québec à Montréal

Wilson, Adam. 2016. Les dynamiques sociolinguistiques de la globalisation : l'exemple de l'office du Tourisme de Marseille, thèse de doctorat, Université Aix-Marseille

Yanoshevsky, Galia. 2017. «Le photobook comme guide touristique : le cas d'À travers Israël (1950-1951) ». Raus, Rachele, Gloria Cappelli \& Carolina Flinz (éds), Le guide touristique: lieu de rencontre entre lexique et images du patrimoine culturel, vol. 2, 157-186 (Florence: Firenze U. P.)

Yanoshevsky, Galia. 2020. «L'identité de l'Un dans le regard de l'Autre : Israël dans les guides touristiques et la question du locuteur collectif », Paissa, Paola \& Roselyne Koren (éds). Du singulier au collectif: construction(s) discursive(s) de l'identité collective dans le débat public (Limoges : Lambert-Lucas), 129-147

\section{NOTES}

1. Ce numéro est le résultat d'un atelier de recherche subventionné par la Israel Science Foundation (ISF) qui s'est déroulé en février 2019 et qui portait sur les Discourses and Materialities of Tourism.

2. Je m'associe ici à Dominique Maingueneau, qui dans un article qui réhabilite la notion de "formation discursive», dit que "les chercheurs ont besoin d'associer des matériaux hétérogènes sans les ramener à l'unité, mais sans non plus ignorer leurs multiples interactions » (Maingueneau 2011: 96).

3. Cette idée de motivation idéologique qui régit l'activité touristique se manifeste aussi dans d'autres études, par ex. chez Cohen-Hattab (2004), qui voit un lien étroit entre l'image propagée d'un site touristique et les forces idéologiques qui contrôlent l'industrie touristique de l'État (les voyagistes, les tours guidés, la presse et les guides touristiques).

4. En 2011 encore, Moïse déplore la rareté des études sociolinguistiques - entendues dans le sens de sociolinguistique, ethnographique et interactionnelle - qui tentent de comprendre, à travers l'analyse des discours et des interactions langagières, les processus et les changements sociaux.

5. Sur l'aspect du stéréotypage et des lieux communs du tourisme, bien que dans une perspective plutôt géographique, voir le travail de l'équipe MIT (2002).

6. À partir d'une étude sémiotique de corpus : de guides généralistes présentant New York et l'Écosse (Le Routard, les guides Bleu, Vert et Voir, le Lonely Planet, le Petit Futé et la Bibliothèque du voyageur) mais aussi de guides "spécialisés " (Guide du Paris mystérieux, Le Guide de la France mythologique et les Sites mystérieux et légendaires de nos provinces françaises).

7. « En valorisant et en mettant en discours un certain espace touristique, le guide s'inscrit dans une époque et dans une culture donnée. Les professionnels de la communication touristique parlent alors de "mise en tourisme de la destination" »(Seoane 2013 : par. 3).

8. «Qu'est-ce que le texte? Partons de la définition suivante: il s'agit d'une configuration signifiante d'objets sémiotiques inscrite sur un support matériel et prise dans un contexte de communication », Souchier et Wrona $2015: 174)$.

9. «Il s'agit d'analyser la dimension textuelle des phénomènes de communication. L'approche épistémologique ici mise en œuvre consiste à travailler sur la relation qu'entretiennent "pensée du texte" et "pensée de la communication". Une relation qui tire parti des apports de la théorie du texte hérités de la linguistique, de la littérature ou de la sémiologie et les conjugue à une préoccupation communicationnelle tenant compte tout à la fois de la forme des échanges, de la 
dimension contextuelle du processus de production ou de la prise en compte des techniques d'écriture et de lecture, notamment » (Souchier et Wrona :173-174).

10. A ce sujet cf. aussi l'étude critique de l'équipe MIT (2002), qui vise à critiquer la vision négative du touriste et du tourisme à partir de l'analyse des lieux communs et des espaces géographiques propres au tourisme.

11. On notera en passant le Manuel de l'anti-tourisme de Rodolphe (2017), une dénonciation des méfaits du tourisme sur l'équilibre écologique de la planète.

12. https://www.lhistoire.fr/le-tourisme-stade-ultime-du-colonialisme

\section{AUTHOR}

\section{GALIA YANOSHEVSKY}

Université Bar-Ilan, ADARR 